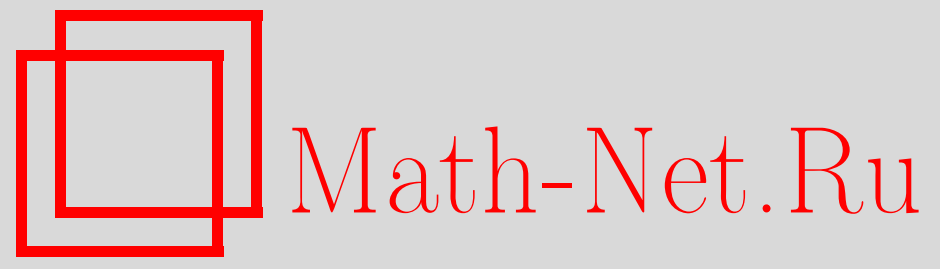

Д. Н. Бабин, Конечность множества автоматных базисов Поста с разрешимой проблемой полноты, Дискрет. матем., 1998, том 10, выпуск 3, 57-63

DOI: https://doi.org/10.4213/dm430

Использование Общероссийского математического портала Math-Net.Ru подразумевает, что вы прочитали и согласны с пользовательским соглашением http://www.mathnet.ru/rus/agreement

Параметры загрузки:

IP : 52.6 .47 .48

26 апреля 2023 г., $16: 38: 14$

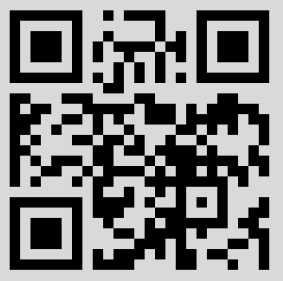




\title{
Конечность множества автоматных базисов Поста с разрешимой проблемой полноты
}

(C) 1998 г. Д. Н. Бабин

\begin{abstract}
Рассматриваются базисы вида $M=\Phi \cup \nu$, где $\Phi$ - некоторый класс Поста, а $\nu$ - конечная система автоматных функций. Показано, что множества классов Поста $\Phi$, для которых разрешима проблема полноты $\Phi \cup \nu$, соответственно, $A$ полноты $\Phi \cup \nu$, являются конечными.
\end{abstract}

\section{1. Введение}

Для базисов автоматных функций без памяти (булевых функций) проблема полноты относительно суперпозиции была решена Постом [1]. При обобщении этой задачи на автоматные функции с памятью при использовании суперпозиции и обратной связи возникли существенные трудности. Принципиальная невозможность решения этой задачи в общем случае была установлена в [2], где была показана континуальность всякой критериальной системы, а в [3] установлена ее алгоритмическая неразрешимость для конечных базисов автоматных функций. Тем не менее, для базисов, содержащих все булевы функции, указанная задача алгоритмически разрешима [4]. Проблема $A$-полноты для систем автоматных функций также алгоритмичски неразрешима в общей постановке [5] и разрешима для систем, содержащих все булевы функции [6]. Естественно исследовать на полноту и $A$-полноту системы Поста вида $M=\Phi \cup \nu$, где $\Phi$ - некоторый класс Поста, а $\nu$ - конечная система автоматных функций. Возникает разделение классов Поста на сильные и слабые по их способности гарантировать разрешимость полноты и $A$-полноты для автоматных функций. В данной работе показано, что множество сильных классов Поста конечно.

\section{2. Основные понятия и результаты}

Мы будем использовать обозначения из $[7,8]$. Пусть $E_{2}=\{0,1\}, E_{2}^{\infty}-$ множество всех сверхслов $a(1) a(2) \ldots$, где $a(j) \in E_{2}, j=1,2, \ldots$, и $E_{2}^{\tau}-$ множество всех слов $a(1) \ldots a(\tau)$ длины $\tau$. Пусть $f:\left(E_{2}^{\infty}\right)^{n} \rightarrow\left(E_{2}^{\infty}\right)^{m}$ - автоматная функция 
(а.-функция), то есть функция, которая задается рекуррентно соотношениями

$$
\begin{aligned}
q(1) & =q_{1}, \\
q(t+1) & =\varphi\left(q(t), a_{1}(t), \ldots, a_{n}(t)\right), \\
b_{j}(t) & =\psi_{j}\left(q(t), a_{1}(t), \ldots, a_{n}(t)\right),
\end{aligned}
$$

где $q \in Q=\left\{q_{1}, \ldots, q_{r}\right\}$ и $j=1, \ldots, m$. Параметр $q$ называется состоянием а.-функции $f, q_{1}$ - ее начальным состоянием, вектор-буквы $a=\left(a_{1}, \ldots, a_{n}\right)$ и $b=\left(b_{1}, \ldots, b_{m}\right)$ называются входной и выходной буквами, а сверхслова $a(1) a(2) \ldots$ и $b(1) b(2) \ldots$ - входным и выходным сверхсловами соответственно.

Класс всех а.-функций обозначим через $P$. В этом классе обычным образом введем операции суперпозиции и обратной связи, которые далее будем интерпретировать на схемном языке. Пусть $M \subseteq P$, обозначим через $[M]$ множество всех а.-функций, получающихся из $M$ с помощью операций суперпозиции и обратной связи. Множество $M$ называется полным, если $[M]=P$. Проблема полноты для $P$ состоит в описании всех полных множеств $M$.

Пусть $\tau$ - натуральное число, $f\left(x_{1}, \ldots, x_{n}\right)$ - некоторая автоматная функция, $f^{\tau}:\left(E_{2}^{\tau}\right)^{n} \rightarrow\left(E_{2}^{\tau}\right)^{m}$ - ограничение этой функции на множество слов длины $\tau$. Скажем, что а.-функции $f\left(x_{1}, \ldots, x_{n}\right)$ и $g\left(x_{1}, \ldots, x_{n}\right) \tau$-равны, если $f^{\tau}=g^{\tau}$. Обозначим через $[M]_{\tau}$ множество всех а.-функций, $\tau$-равных получающимся из $M$ с помощью операций суперпозиции и обратной связи (о.с.). Известно [8], что результат применения о.с. $\tau$-равен $\tau$ применениям суперпозиции. Множество $M$ называется $\tau$-полным, если $[M]_{\tau}=P$. Множество $M$ называется $A$-полным, если $[M]_{\tau}=P$ при всех $\tau$. Пусть

$$
[M]_{A}=\bigcap_{\tau}[M]_{\tau}
$$

Проблема $A$-полноты для $P$ состоит в описании всех $A$-полных множеств $M$. Очевидно, что полное множество $M$ является $A$-полным. Обозначим через $P^{(1)}$ класс всех а.-функций с одним состоянием. $A$-функции из $P^{(1)}$ называются истинностными и могут рассматриваться как булевы функции. $A$-функция $B: E_{2}^{\infty} \rightarrow E_{2}^{\infty}$ с уравнениями

$$
q(1)=1, \quad q(t+1)=x(t), \quad b(t)=q(t)
$$

называется а.-функцией задержки. В $P$ имеются конечные полные системы. Известно, например, что $\left[\left\{B, f_{\mathrm{Sh}}\right\}\right]=P$, где $f_{\mathrm{Sh}}-$ универсальная булева функция Шеффера.

Замкнутые классы булевых функций полностью описаны Постом $[1,7]$. Все они конечнопорожденные и образуют счетную решетку по включению. Автор показал в [4], что для верхнего элемента решетки, класса всех булевых функций $\Phi=P^{(1)}=\left[f_{\mathrm{Sh}}\right]$ имеется алгоритм определения полноты системы $M=\Phi \cup \nu$. Пусть $\pi$ - множество всех классов Поста, Sol и $\operatorname{Sol}_{A}-$ множества таких $\Phi \in \pi$, что для конечных $\nu \subset P$ проблема полноты $\Phi \cup \nu$ и, соответственно, $A$-полноты $\Phi \cup \nu$ разрешима, a UnSol и $\mathrm{UnSol}_{A}$ суть аналогичные множества с неразрешимой проблемой полноты. Назовем классы из $\mathrm{Sol}_{\text {и }} \mathrm{Sol}_{A}$ сильными классами Поста, а из $\mathrm{UnSol} \mathrm{и} \mathrm{UnSol}_{A}$, соответственно, слабыми. Заметим, что всякий надкласс сильного класса является сильным, а подкласс слабого слабым, двойственный к сильному, относительно замены 0 на 1 , будет сильным, а к слабому слабым классом Поста. Пусть

$$
h\left(x_{1}, x_{2}, x_{3}, x_{4}\right)=x_{1} x_{2} \vee x_{1} x_{3} \vee x_{1} x_{4} \vee x_{2} x_{3} \vee x_{2} x_{4} \vee x_{3} x_{4}
$$


$F=[\{h, \bar{x} \vee y\}]$ и $F^{\prime}-$ класс, двойственный к $F$. Имеет место следующая теорема.

Теорема 1. Проблемы полноты и А-полноты для каждого из классов базисов Поста вида $F \cup \nu$ или $F^{\prime} \cup \nu$ алгоритмически неразрешимы.

Известно [1, 7], что число классов $\Phi \in \pi$, содержащихся в $F^{\prime}$ или в $F$ бесконечно, а не содержащихся ни в $F^{\prime}$, ни в $F$ конечно, поэтому имеет место следующее утверждение.

Следствие 1. Множества $\mathrm{Sol} u \mathrm{Sol}_{A}$ конечны, множества UnSol $u \mathrm{UnSol}_{A}$ бесконечны.

\section{3. Основные леммы}

Тройка $\Theta=\langle D, \rho, w\rangle$, где $D=\left\{d_{1}, \ldots, d_{k}\right\}, D^{*}$ - множество слов в алфавите $D$, $\rho: D \rightarrow D^{*}, \rho\left(d_{i}\right)=R_{i}, w$ - натуральное число, называется системой однородных продукций Поста. Если $l \geqslant w$, то скажем, что $\Theta$ применима к слову $\xi=d_{i_{1}} d_{i_{2}} \ldots d_{i_{l}}$ и слово $\Theta(\xi)=d_{i_{w+1}} d_{i_{w+2}} \ldots d_{i_{l}} R_{i_{1}}$ назовем результатом применения $\Theta$ к слову $\xi$. Последовательность $\xi_{1}, \xi_{2} \ldots$ такую, что $\xi_{1}=\xi$, а $\xi_{i+1}=\Theta\left(\xi_{i}\right)$, назовем последовательностью продукций слова $\xi$. Известно [10], что существует система однородных продукций Поста, для которой не существует алгоритма, по слову $\xi$ решающего вопрос о конечности последовательности продукций слова $\xi$. Зафиксируем систему продукций Поста $\langle D, \rho, w\rangle$ с неразрешимой проблемой конечности последовательности продукций.

Обозначим через $R$ множество сверхслов $\alpha$ таких, что $\alpha(t)=1$ при $t>t_{\alpha}$. Пусть $\mathbf{1}, 01,0011$ суть периодические сверхслова $11 \ldots, 010101 \ldots, 00110011 \ldots ; \alpha]_{t}-$ слово $a(1) \ldots a(t)$, где $\alpha=a(1) a(2) \ldots ; \mathbf{N}-$ множество натуральных чисел; $\mathbf{N}_{0}-$ множество $\mathbf{N} \cup\{0\} ;|\alpha|$ - длина слова $\alpha$; если $d_{i} \in D$, то $\tilde{d}_{i}-$ слово из $E_{2}^{(4 k+4)}$ вида $\tilde{d}_{i}=(0110)^{i}(0101)(0110)^{k-i}$; если $\xi=d_{i_{1}} d_{i_{2}} \ldots d_{i_{l}}$, то $\tilde{\xi}=\tilde{d}_{i_{1}} \tilde{d}_{i_{2}} \ldots \tilde{d}_{i_{l}}$. Пусть

$$
\begin{aligned}
& M_{i}=\left\{(0101)^{n} \tilde{d}_{i} \tilde{d}_{j_{1}} \ldots \tilde{d}_{j_{l}} \mathbf{0 0 1 1} \mid n, l \in \mathbf{N}_{0}, 1 \leqslant j_{1}, \ldots, j_{l} \leqslant k\right\}, \\
& \hat{M}_{i}=\left\{(0101)^{n} \tilde{d}_{i} \tilde{d}_{j_{1}} \tilde{d}_{j_{2}} \ldots \mid n \in \mathbf{N}_{0}, 1 \leqslant j_{1}, j_{2} \ldots \leqslant k\right\} .
\end{aligned}
$$

Для $\alpha, \beta \in E_{2}^{\infty}, \alpha \neq \beta$, положим

$$
\left.\left.c(\alpha, \beta)=\max \{t \mid \alpha]_{t}=\beta\right]_{t}\right\}
$$

и для $\alpha \notin M_{i} \cup \hat{M}_{i}$

$$
c_{i}(\alpha)=\max \left\{c(\alpha, \beta) \mid \beta \in M_{i} \cup \hat{M}_{i}\right\}
$$

Определим а.-функции $f_{i}: E_{2}^{\infty} \rightarrow E_{2}^{\infty}, f_{i}^{\prime}: E_{2}^{\infty} \rightarrow E_{2}^{\infty}, i=1,2, \ldots, k$, полагая

$$
\begin{aligned}
& f_{i}\left((0101)^{n} \tilde{d}_{i} \tilde{d}_{j_{1}} \ldots \tilde{d}_{j_{l}} \mathbf{0 0 1 1}\right)= \begin{cases}(0101)^{n+w(k+1)} \tilde{d}_{j_{w}} \ldots \tilde{d}_{j_{l}} \tilde{R}_{i} \mathbf{0 0 1 1}, & l>w-1, \\
(0101)^{n+w(k+1)} \tilde{R}_{i} \mathbf{0 0 1 1}, & l=w-1, \\
\mathbf{0 1}, & l<w-1,\end{cases} \\
& f_{i}^{\prime}\left((0101)^{n} \tilde{d}_{i} \tilde{d}_{j_{1}} \ldots \tilde{d}_{j_{l}} \mathbf{0 0 1 1}\right.= \begin{cases}(0101)^{n+w(k+1)} \tilde{d}_{j_{w}} \ldots \tilde{d}_{j_{l}} \tilde{R}_{i} \mathbf{0 0 1 1}, & l>w-1, \\
(0101)^{n+(l+1)(k+1)} 0 \mathbf{1}, & l \leqslant w-1,\end{cases} \\
& f_{i}(\beta)=\gamma \mathbf{1}, \quad f_{i}^{\prime}(\beta)=\gamma^{\prime} \mathbf{1}, \quad \beta \notin M_{i} \cup \hat{M}_{i}, \quad\left|\gamma^{\prime}\right|=|\gamma|=c_{i}(\beta) .
\end{aligned}
$$


Для $\alpha, \gamma \in E_{2}^{\infty}$ определим а.-функции $G, E, f_{\xi}$, полагая

$$
\begin{aligned}
G(\alpha, \gamma) & = \begin{cases}B(\alpha), & \gamma=\mathbf{0 1}, \\
\delta \mathbf{1}, & \gamma \neq \mathbf{0 1},\end{cases} \\
E(\gamma) & = \begin{cases}00 \ldots, & \gamma=\mathbf{0 1}, \\
\beta \mathbf{1}, & \gamma \neq \mathbf{0 1},\end{cases} \\
f_{\xi}(\alpha) & =\tilde{\xi} \mathbf{0 1}, \quad \alpha \in E_{2}^{\infty},
\end{aligned}
$$

где $|\delta|=|\beta|=c(\gamma, \mathbf{0 1})$.

Через $\bar{X} \vee Y, H, \mathbf{0}, X \vee Y, X Y$ обозначим а.-функции, являющиеся автоматными интерпретациями булевых функций $\bar{x} \vee y, h, 0, x \vee y, x y$, соответственно. Справедливы следующие леммы.

Лемма 1. Система $\Sigma=\left\{\bar{X} \vee Y, H, E, f_{1}, \ldots, f_{k}, f_{\xi}, G\right\}$ полна тогда и толъко тогда, когда последовательность продукиий слова $\xi$ конечна.

Лемма 2. Система $\Sigma^{\prime}=\left\{\bar{X} \vee Y, H, E, f_{1}{ }^{\prime}, \ldots, f_{k}{ }^{\prime}, f_{\xi}, G\right\}$-полна тогда и только тогда, когда последовательность продукций слова $\xi$ бесконечна.

\section{4. Доказательства лемм}

Через $\hat{H}$ обозначим покомпонентное доопределение функции $h$ на $\left(E_{2}^{4}\right)^{4}$. Для $\alpha_{1}$, $\alpha_{2}$ из $E_{2}^{\infty}$ будем писать $\alpha_{1} \geqslant \alpha_{2}$, если $\alpha_{1}(t) \geqslant \alpha_{2}(t)$ при всех $t$. Пусть $\xi_{1}, \xi_{2}, \ldots, \xi_{s}$ - некоторые последовательные продукции слова $\xi=\xi_{1}$ и

$$
\begin{aligned}
\eta_{1} & =\tilde{\xi}_{1} \mathbf{0 0 1 1}, \\
\eta_{i} & =(0101)^{w(i-1)(k+1)} \tilde{\xi}_{i} \mathbf{0 0 1 1}, \quad i=2,3, \ldots, s .
\end{aligned}
$$

Пусть $b_{i}=1+4 w(k+1)(i-1)$ и $t_{i}=b_{i}+\left|\xi_{i}\right|-1$ - позиции, соответственно, начала и конца (включительно) подслова $\tilde{\xi}_{i}$ в сверхслове $\eta_{i}$. Если $b_{i+1}<t_{i}$, то $\eta_{i}(t)=\eta_{i+1}(t)$ при $b_{i+1} \leqslant t \leqslant t_{i}$. Имеет место следующее утверждение.

Предложение 1. Если $\alpha_{0}, \alpha_{1}, \alpha_{2}, \alpha_{3} \in E_{2}^{\infty} u$ nрu $i \leqslant p \leqslant r$

$$
\alpha_{1} \geqslant \eta_{i}, \quad \alpha_{2} \geqslant \eta_{p}, \quad \alpha_{3} \geqslant \eta_{r},
$$

mo $H\left(\alpha_{0}, \alpha_{1}, \alpha_{2}, \alpha_{3}\right) \geqslant \eta_{p}$.

Доказательство. Ввиду монотонности функции $h$

$$
\gamma=H\left(\alpha_{0}, \alpha_{1}, \alpha_{2}, \alpha_{3}\right) \geqslant \eta_{i} \eta_{p} \vee \eta_{i} \eta_{r} \vee \eta_{p} \eta_{r}
$$

Пусть $b_{i} \leqslant b_{p}<t_{i}<b_{r}<t_{p} \leqslant t_{r}$. Для $t \in\left(t_{i}, b_{r}-3\right)$

$$
(\gamma(t), \gamma(t+1), \gamma(t+2), \gamma(t+3))=\hat{H}(\cdot, 0011, \Delta, 0101) \geqslant \Delta
$$

где $\Delta=\left(\eta_{p}(t), \eta_{p}(t+1), \eta_{p}(t+2), \eta_{p}(t+3)\right)$ и $\Delta \in\{0110,0101\}$. Для $t \notin\left(t_{i}, b_{r}-3\right)$ либо $\eta_{p}(t)=\eta_{r}(t)$, либо $\eta_{p}(t)=\eta_{i}(t)$, следовательно, $\gamma(t) \geqslant \eta_{p}(t)$. В остальных случаях взаимного расположения чисел $b_{i}, b_{p}, b_{r}, t_{i}, t_{p}, t_{r}$ возникают аналогичные ситуации. Предложение доказано. 
Доказательство леммы 1. Начнем с доказательства необходимости. Пусть система продукций $\xi_{1}, \xi_{2}, \ldots$ бесконечна и система $\Sigma$ при этом полна. Тогда найдется схема $S$ над $\Sigma$, реализующая константную а.-функцию $C_{01}$ с выходным словом 01. Можно считать, что схема $S$ получилась из $\Sigma$ применением сначала всех операций суперпозиции, а потом всех операций о.с. Обозначим через $S_{t}$ схему, получающуюся из $S$ заменой ее а.-функций булевыми функциями их состояний в момент $4 t+1$. Заметим, что а.-функции $E, f_{1}, \ldots, f_{k}$ будут заменены на функцию $X$ или на $\mathbf{1}$; $f_{\xi}$ будет заменена на $0 ; G$ на 1 или $Y$. Каждая из схем $S_{t}$ реализует константу 0. Пусть $g$ - элемент схемы $S_{t}$, выход которого совпадает с выходом схемы. Разорвем обратную связь от $g$ (если она имеется) в схеме $S_{t}$ и подставим на образовавшийся вход константу $\mathbf{1}$, в результате новая схема $S_{t}{ }^{\prime}$ также будет давать константу 0. Возможны следующие варианты :

(a) $g$ - выходная функция состояния одного из элементов $E, f_{1}, \ldots, f_{k}$, то есть $g \equiv x$, а $S_{t}{ }^{\prime}=g\left(S^{\prime}\right)$, и, следовательно, $S^{\prime}$ реализует константу 0.

(b) $g$ - это функция $\bar{X}_{1} \vee X_{2}$, тогда $\bar{x}_{1} \vee x_{2} \equiv 0$, значит, $x_{2} \equiv 0$; для $g=G$ также $x_{2} \equiv 0$ (случай, когда элемент $g=G$ имеет в момент $4 t+1$ состояние с выходной функцией 1 невозможен). Получаем схему $S^{\prime}$ с двумя выходами, имеющую на правом выходе 0 , такую что $S_{t}^{\prime}=g\left(S^{\prime}\right)$.

(c) Для $g=h$ имеем $h\left(x_{1}, x_{2}, x_{3}, x_{4}\right) \equiv 0$, что с точностью до перестановки $x_{1}, x_{2}$, $x_{3}, x_{4}$ дает $x_{2} \equiv x_{3} \equiv x_{4} \equiv 0$. Получается схема $S^{\prime}$ с тремя нулевыми выходами такая, что $S_{t}^{\prime}=g\left(S^{\prime}\right)$. Заметим, что в исходной схеме $S$ на каждом из этих выходов не может быть константы 1.

Пометим элемент $g$, пометим его нулевой вход (входы) и рассмотрим те элементы схемы $S^{\prime}$, выходы которых совпадают с выходами самой схемы $S^{\prime}$. Проделаем с ним подходящее действие (a), (b) или (c). Эти элементы не могли быть ранее помечены, так как обратные связи от ранее помеченных элементов разорваны. Продолжая этот процесс, пометим в схеме $S_{t}$ дерево, вершины которого суть помеченные элементы, а ребра помеченные входы элементов схемы $S_{t}$. Листьями дерева будут являться элементы 0.

Перенеся метки в схему $S$, получим в ней дерево $T_{t}$. Листья дерева $T_{t}$ суть элементы вида $f_{\xi}$; в вершины вида $E, f_{1}, \ldots, f_{k}$ входит одно ребро, в вершины вида $\bar{X}_{1} \vee X_{2}, G$ входит также одно ребро, соответствующее их правому входу, в вершины вида $H$ входят три ребра. При этом в схеме $S$ на каждом из выходов вершин дерева $T_{t}$ не может быть константы 1 . Из каждой вершины дерева $T_{t}$ выходит одно ребро. Последовательность деревьев $T_{1}, T_{2}, \ldots$ периодическая $\left(T_{t}\right.$ зависит от состояния $q(t)$ схемы $S$, не имеющей входа). Пусть $T_{r}, \ldots T_{r+\not{K}}$ встречаются в последовательности $T_{1}, T_{2}, \ldots$ бесконечно часто. Рассмотрим дерево $T$, множества вершин и, соответственно, ребер которого суть объединения вершин и ребер деревьев $T_{r}, \ldots T_{r+K}$. (В дереве $T$ возможна вершина, элемент $H$, в которую входит четыре ребра). Пусть

$$
L_{1}=\bigcup_{i}\left\{\alpha \mid \alpha \geqslant \eta_{i}\right\}, \quad L=L_{1} \cup R .
$$

При всех $i=1,2, \ldots, k$

$$
\begin{gathered}
f_{i}: L_{1} \rightarrow L_{1}, \quad f_{i}: R \rightarrow R, \quad E: L \rightarrow R, \quad G: E_{2}^{\infty} \times L \rightarrow R, \\
\bar{X}_{1} \vee X_{2}: E_{2}^{\infty} \times L_{1} \rightarrow L_{1}, \quad \bar{X}_{1} \vee X_{2}: E_{2}^{\infty} \times R \rightarrow R .
\end{gathered}
$$


В предложении 1 было показано, что $H: E_{2}^{\infty} \times L_{1} \times L_{1} \times L_{1} \rightarrow L_{1}$. Очевидно, что $H: E_{2}^{\infty} \times E_{2}^{\infty} \times R \times R \rightarrow R$. Покажем, что в схеме $S$ не может быть случая, когда на элемент $H$, имеющий в дереве $T$ три входящих ребра, поступят последовательности $\alpha_{1} \in R$ и $\alpha_{2}, \alpha_{3} \in L_{1}$. В самом деле, пусть $\alpha_{1}=\alpha 1,|\alpha|=t_{0}$, тогда существует $t>t_{0}$ такое, что в схеме $S_{t}{ }^{\prime}$ при построении дерева $T_{t}{ }^{\prime}$ возникнет этот элемент $H$, имеющий три нуля на рассматриваемых входах, что противоречит тому, что при $t>t_{0}$ будет $\alpha_{1}(t)=1$. Значит, этот элемент $H$ имеет четыре ребра в дереве $T$. Таким образом, на выходе схемы $S$ должно получиться сверхслово из $L$, получаем противоречие.

Докажем теперь достаточность. Если последовательность продукций слова $\xi$ конечна и имеет вид $\xi_{1}, \xi_{2}, \ldots \xi_{s}$, a $d_{i_{p}}$ - первая буква в $\xi_{p}$ и $\left|\xi_{s}\right|<w$, то

$$
f_{i_{s}}\left(f_{i_{s-1}} \ldots f_{i_{1}}\left(f_{\xi}\right) \ldots\right)=C_{01}, \quad E(\mathbf{0 1})=\mathbf{0}, \quad G(x, \mathbf{0 1})=B(x) .
$$

Следовательно, $\Sigma$ - полная система. Лемма 1 доказана.

Доказательство леммы 2. Начнем с доказательства необходимости. Предположим противное. Пусть система $\Sigma^{\prime}$ полна, а последовательность продукций $\xi$ конечна и равна $\xi_{1}, \xi_{2}, \ldots, \xi_{s}$. Пусть

$$
\begin{gathered}
\tau=4 w(k+1) s+5, \quad \eta_{1}=\tilde{\xi}_{1} \mathbf{0 0 1 1} \\
\eta_{i}=(0101)^{w(i-1)(k+1)} \tilde{\xi}_{i} \mathbf{0 0 1 1}, \quad \eta_{s+1}=(0101)^{w s(k+1)} 0 \mathbf{1}
\end{gathered}
$$

$\eta_{i+1}=f_{j_{i}}\left(\eta_{i}\right)$ при подходящем $j_{i}, i=1, \ldots, s$,

$$
L_{1}=\bigcup_{i}^{s}\left\{\alpha \mid \alpha \geqslant \eta_{i}\right\}, \quad L_{2}=\left\{\alpha^{\prime} \mathbf{1}|| \alpha^{\prime} \mid=\tau-1\right\} .
$$

Пусть схема $S$ над $\Sigma^{\prime}$ реализует константу $0101 \ldots 010$ длины $\tau$. Без ограничения общности в схеме $S$ нет обратных связей и все операции отождествления входов выполнены в последнюю очередь. Очевидно, что $f_{\xi}$ присутвует в схеме $S$, так как булевы функции начальных состояний остальных элементов $\Sigma^{\prime}$ сохраняют единицы. Подставим $f_{\xi}$ на фиктивные входы схемы $S$ и получим схему $S^{\prime}\left(f_{\xi}\right)$, где схема $S^{\prime}$ элементов $f_{\xi}$ не содержит. На выходе $f_{\xi}$ получилась константа $\left.\tilde{\xi}_{1} \mathbf{0 0 1 1}\right]_{\tau}$ из $L_{1}$. Покажем, что все а.-функции $g$ из $\Sigma^{\prime} \backslash\left\{f_{\xi}\right\}$ сохраняют $L_{1} \cup L_{2}$. В самом деле, при всех $i=1,2, \ldots, k$

$$
\begin{aligned}
f_{i}^{\prime}: L_{1} \rightarrow L_{1} \cup L_{2}, & f_{i}^{\prime}: L_{2} \rightarrow L_{2}, \quad E: L_{1} \cup L_{2} \rightarrow L_{2}, \\
G: E_{2}^{\infty} \times\left(L_{1} \cup L_{2}\right) \rightarrow L_{2}, & \bar{X}_{1} \vee X_{2}: E_{2}^{\infty} \times\left(L_{1} \cup L_{2}\right) \rightarrow\left(L_{1} \cup L_{2}\right) .
\end{aligned}
$$

Покажем, что $H:\left(L_{1} \cup L_{2}\right)^{4} \rightarrow\left(L_{1} \cup L_{2}\right)$. В самом деле, если три из четырех входных слов $\alpha_{0}, \alpha_{1}, \alpha_{2}, \alpha_{3}$ из $L_{1}$, то по предложению $1 H\left(\alpha_{0}, \alpha_{1}, \alpha_{2}, \alpha_{3}\right) \in L_{1}$, в альтернативном случае два из них принадлежат $L_{2}$, что приводит к тому, что $H\left(\alpha_{0}, \alpha_{1}, \alpha_{2}, \alpha_{3}\right) \in L_{2}$. Таким образом, на выходе схемы $S$ получится слово из $L_{1} \cup L_{2}$, получаем противоречие.

Докажем теперь достаточность. Если последовательность продукций слова $\xi$ бесконечна и имеет вид $\xi_{1}, \xi_{2}, \ldots$, а $d_{i_{p}}$ - первая буква $\xi_{p}$, то на словах длины $\tau$

$$
f_{i_{\tau}}\left(f_{i_{\tau-1}} \ldots f_{i_{1}}\left(f_{\xi}\right) \ldots\right)=C_{\mathbf{0 1}}, \quad E(\mathbf{0 1})=\mathbf{0}, \quad G(x, 01)=B(x) .
$$

Следовательно, $\Sigma^{\prime}$ есть $A$-полная система. Лемма 2 доказана. 
Автор выражает благодарность В. Б. Кудрявцеву за постановку задачи и ценные указания.

\section{Список литературы}

1. Post E. Two-Valued Iterative Systems of Mathematical Logic. Princeton Univ. Press, Princeton, 1941.

2. Кудрявцев В. Б. О мощностях множеств предполных классов некоторых функциональных систем, связанных с автоматами. Докл. АН СССР (1963) 151, №3, 493-496.

3. Кратко М. И. Алгоритмическая неразрешимость проблемы распознавания полноты для конечных автоматов. Докл. АН CCCP (1964) 155, №1, 35-37.

4. Бабин Д. Н. Разрешимый случай задачи о полноте автоматных функций. Дискретная математика (1992) 4, №4, 41-55.

5. Буевич В. А. Об алгоритмической неразрешимости распознавания $A$-полноты. Матем. заметки (1972) 12, №6, 687-697.

6. Буевич В. А. Условия А-полноты для конечных автоматов. Изд. МГУ, Москва, 1986.

7. Кудрявцев В. Б., Гаврилов Г. П., Яблонский С. В. Функции алгебры логики и классъ Поста. Наука, Москва, 1966.

8. Кудрявцев В. Б., Алешин С. В., Подколзин А. С. Введение в теорию автоматов. Наука, Москва, 1985.

9. Мальцев А. И. Итеративные алгебры Поста. ИМ СО АН СССР, Новосибирск, 1976.

10. Мальцев А. И. Алгоритмы и рекурсивные функции. Наука, Москва, 1986.

Статья поступила 18.05.1998. 